\title{
Obesity: does a one-size intervention fit all?
}

\author{
Rachel Ashton
}

The US and UK obesity epidemics continue to spiral out of control. Since only the mid-1970s the number of overweight and obese adults in the US, according to the FDA, has more than doubled, and that of obese children ( $>6$ years) has risen roughly threefold. The Health Profile of England 2007 government report states that in 1995-2005 the proportions of obese women, men and children rose by almost $35 \%,>40 \%$, and $50 \%$, respectively.

Much of a generation of adults in the US and the UK now barely remembers a lifestyle before comfortable armchairs, a dazzling array of convenience foods and gadgets, and the rise of the desk-occupying service industry. Although these factors have contributed substantially to causing us harm, trying to persuade people to live life differently from the way they know is a major challenge.

In 2004, the FDA launched its Calories Count campaign to help people reduce weight through balancing calorie intake with calorie expenditure. Labeling of ingredients and nutrition has become more informative, online software has been published to help calculate calorie intake and portion sizes, and partnerships have been formed with various companies to help educate adults and children about food.

In the UK, the Food Standards Agency introduced a 'traffic light' food labeling system to help consumers identify at a glance levels of fat, saturated fat, salt and sugar in prepackaged foods (red high, amber medium, green low). The Department of Health has also launched Healthy Weight, Healthy Lives, which is a cross-department strategy aimed at promoting healthier food choices, children's health and activity, and at providing information for people who are already overweight or obese.
"How can

information

on so complex

an issue be

correctly

pitched to such

a broad range

of recipients?"

$R$ Ashton is the Associate Publisher of Nature Clinical Practice.

\section{Competing interests}

The author declared no competing interests.

www.nature.com/clinicalpractice doi:10.1038/ncpendmet0862
Although the schemes do include some efforts to provide facilities and training that will improve access to active past-times and improve fitness, much of the interventions rely on the imparting of information. Is information enough? Irrespective of whether it reaches the people who need it most, many factors are involved in who is interested and how it is processed-for example, age, level of education, cultural origins, socioeconomic status, and so on. How can information on so complex an issue be correctly pitched to such a broad range of recipients?

Take food labeling as an example. Displaying nutritional data, portion sizes and proportions of recommended daily amounts, as well as assigning 'goodness' and 'badness', are all very well. The fact remains, however, that foods high in fats, salt and sugar are still being widely bought. Could people be unaware of the meanings of the labels or ignoring them? Could they just not care if the food is something they enjoy eating? Irrespective of personal choice, low income and/or lack of time can be major barriers to buying fresh, healthy ingredients; information will not increase money and time. Thus, foods with low nutritional and, seemingly, monetary values that provide a high convenience value will continue to sell.

Governments in the US and UK certainly seem to be grasping the public-health gauntlet of obesity. Who will truly really benefit is, however, unclear. Although they can be seen to be doing the right things, the people who will listen with the keenest ears are probably already seeking out the information. These governments could, therefore, merely be preaching to the converted. For the rest of the consumers, a one-size intervention at the general population level might not fit all. 